\title{
PROCESO DE INTERNACIONALIZACIÓN DE EMPRESAS DEL ÁREA METROPOLITANA DE BUCARAMANGA
}

\author{
INTERNATIONALIZATION PROCESS OF FIRMS IN THE \\ BUCARAMANGA METROPOLITAN AREA
}

Édgar Javier Gómez Parada ${ }^{1}$ Gustavo González Sarmiento²

\begin{abstract}
Resumen
En el presente artículo se muestran los resultados de una investigación sobre el proceso de internacionalización de las empresas manufactureras exportadoras del Área Metropolitana de Bucaramanga (AMB), que toma como referencia la aproximación teórica conocida como el modelo de Uppsala, que considera la situación particular de las PYMES y propone una visión gradualista del proceso. En la primera parte del artículo, se formula un acercamiento a las concepciones mencionadas, para posteriormente presentar los resultados de la investigación y adelantar algunas conclusiones que pueden orientar el diseño de estrategias que mejoren el posicionamiento competitivo de las empresas de la región.
\end{abstract}

\section{Palabras clave}

Internacionalización, PYMES, Internacionalización de Empresas.

\begin{abstract}
The article shows the results of a research conducted in the Internationalization process of manufacturing export companies in the Bucaramanga Metropolitan Area (BMA). The Uppsala theoretical approximation, which considers the particular situation of PYMES and proposes a gradual view of the process, is used as a reference for the purpose of this study. In the first part of the article, the author has an approach to the previously mentioned conceptions to show the results of the research and disclose some conclusions which may guide the design of strategies to improve competitive positioning of companies in the region.
\end{abstract}

\section{Key words}

Internationalization, PYMES, Companies Internationalization

\footnotetext{
1 Ing. Químico (Universidad Industrial de Santander). Mg. en Educación (Universidad Santo Tomás). Docente Facultad de Negocios Internacionales Universidad Santo Tomás. Investigador del Grupo de Investigación de la Facultad de Negocios Internacionales de la Universidad Santo Tomás de Bucaramanga. Áreas de desempeño: Matemáticas, Propiedad intelectual, Competitividad.Email:edjagomezp@yahoo.com

2 Trabajador Social Universidad Industrial de Santander. Especialista en Planeación y Administración del Desarrollo Regional Universidad Cooperativa de Colombia. Docente Facultad de Negocios Internacionales Universidad Santo Tomás. Investigador del Grupo de Investigación de la Facultad de Negocios Internacionales de la Universidad Santo Tomás de Bucaramanga. Áreas de desempeño: Desarrollo regional y competitividad, Globalización y competitividad y Geopolítica. Email: gusta_gsar@yahoo.com
} 


\section{Introducción}

Durante las últimas décadas ha surgido una gran cantidad de literatura que, desde la perspectiva teórica o mediante el registro de resultados de estudios empíricos, ha contribuido a enriquecer el debate sobre los procesos de internacionalización de las empresas que, hace algunos años, se vinculaba únicamente a la expansión de las grandes empresas multinacionales mediante la inversión directa en otros países y hoy se concibe más como un proceso gradual que, a partir de la actividad exportadora simple, puede ser emprendido con éxito por empresas de todos los tamaños. Los temas recurrentes en ese debate tienen que ver con los factores que determinan el éxito de la inserción internacional, la elección del modo de entrada a la competencia internacional y la posibilidad de medir, a través de indicadores, los diferentes niveles de internacionalización.

En ese contexto, el estudio surge del interés de conocer cómo realizan las empresas del Área Metropolitana de Bucaramanga - AMB el proceso de internacionalización y qué percepción tienen los empresarios acerca del mismo. Para los gobiernos locales (Municipio de Bucaramanga y departamento de Santander) y para el nacional, la internacionalización empresarial es una estrategia de desarrollo regional, ante el auge de los tratados internacionales que promueve y concreta el país. La información que resulta de la investigación, sirve para tener un conocimiento sobre la realidad empresarial de la región y sobre los imaginarios individuales y colectivos presentes en el sector productivo respecto a la inserción en mercados externos, que pueden convertirse en motor u obstáculo del proceso.

Existen diferentes concepciones frente a la internacionalización, desde las que la relacionan únicamente con la inserción de las empresas en la dinámica de la economía global hasta aquellas que suponen que el proceso implica el desarrollo de capacidades superiores por parte de las unidades productivas. Desde aquellas que explican tal fenómeno desde el punto de vista de la macroeconomía internacional y del entorno del sector, hasta las que teorizan desde la visión microeconómica de la empresa. Lo cierto es que, para analizar esta tendencia que jalona la definición de estrategias empresariales en la actualidad, se hace necesario apoyarse en un concepto que recoja elementos de estabilidad y permanencia en los mercados internacionales, más cuando se trabaja con empresas medianas y pequeñas, ya que no se puede considerar internacionalización un único y esporádico esfuerzo de exportación. Por ello, coincidimos con Welch y Loustarinen (1988; p 84-85) cuando propone como definición de internacionalización empresarial:

"todo aquel conjunto de operaciones que facilitan el establecimiento de vínculos más o menos estables entre la empresa y los mercados internacionales, a lo largo de un proceso de creciente implicación y proyección internacional de la misma (...) esto es, un proceso amplio y complejo de definición de compromiso internacional adoptado por la organización. Por tanto, se contempla toda una amplia gama o 
variedad de fórmulas institucionales y/o vías de penetración alternativas en el mercado exterior, susceptibles de modificarse en el tiempo, a medida que la empresa va redefiniendo sus niveles relativos de presencia, compromiso de recursos, control y/o riesgo operativo a escala internacional"

Es importante anotar que muchos autores plantean que el proceso de internacionalización de las empresas supone, en la mayoría de los casos, uno paralelo de innovación ya que, para acceder a los mercados externos se requiere normalmente de una redefinición de los objetivos estratégicos de la organización, de reorganizaciones administrativas y reorientación de los programas de mercadeo internacional (Alonso, 1994; Canals, 1996). Incluso, algunos estudios empíricos han demostrado la relación directa entre uno y otro proceso (Simón Carrión, 2010). Este es un tema abierto para la investigación en Colombia, ya que no ha sido abordado de una manera juiciosa ni desde la academia ni desde los estudios que tienen su origen en el sector productivo.

En la línea propuesta por Villarreal (2005) y Galván (2003) se pueden identificar tres grandes enfoques teóricos, que han abordado el análisis de la internacionalización: la teoría del comercio internacional, la de las empresas multinacionales y el enfoque de la internacionalización empresarial propiamente dicho. Según estos autores, la teoría del comercio internacional es de carácter fundamentalmente económico, en tanto que la teoría de las empresas multinacionales es de carácter económicoempresarial. La primera trata de responder a la pregunta: ¿Por qué existe comercio entre los distintos países? en tanto que la segunda se interroga sobre: ¿Por qué existen las empresas multinacionales? (Villarreal 2005).

La teoría del comercio internacional trata de explicar las causas del comercio internacional de bienes y servicios entre países, regiones y sectores económicos, asociadas a la existencia de ventajas comparativas y competitivas. Los enfoques específicos comprendidos dentro de esta teoría, son marcos teóricos relevantes para entender y analizar los determinantes del comercio entre los países o sectores económicos, pero no explican el proceso mismo de la internacionalización a nivel de las Pymes, ni tampoco incorporan variables micro-organizativas presentes dentro del proceso. La teoría de las empresas multinacionales analiza básicamente la internacionalización de las grandes empresas en la forma de la inversión directa en el extranjero y, por lo tanto, excluye la consideración de caminos alternativos seguidos por las de menor tamaño, por lo que tiene un alcance explicativo limitado cuando se trata de esclarecer la situación del sector empresarial colombiano.

Así las cosas, resulta de mucho interés resaltar el debate reciente sobre el tema de la internacionalización, dentro del cual se destacan el enfoque escandinavo, gradualista o modelo de Uppsala, el del ciclo de vida del producto y el enfoque de recursos y capacidades, como los principales modelos explicativos del fenómeno, en especial porque, como afirma Villarreal, quien asume que el proceso compromete toda la cadena de valor, son múltiples las "formas y modalidades que admiten 
estos conceptos" (2005: 3). En el marco de la investigación adelantada sobre el sector exportador del AMB, ese recorrido permitió definir el tipo de indicadores y la perspectiva analítica que más se ajustaba a sus condiciones y confrontar las conclusiones a las de estudios realizados en otros contextos.

\subsection{Enfoque Escandinavo o Modelo de Uppsala}

Este enfoque es uno de los más aceptados en los últimos veinte años para explicar, tanto las razones como las fases de internacionalización de las empresas, especialmente las de menores dimensiones y ha sido referente casi obligado de la gran cantidad de estudios empíricos que se han realizado sobre el tema a nivel internacional. La propuesta parte de trabajos desarrollados en la Escuela Escandinava, por los autores Johanson y Wiedersheim-Paul (1975) y Johanson y Vahlne (1977), en los que se analiza el comportamiento de internacionalización de empresas en Suecia. El interés que despertó la perspectiva de estos autores se explica por el hecho de que son los primeros en reconocer que la ausencia de recursos y de conocimientos sobre los mercados exteriores, genera mucha incertidumbre en las organizaciones y que ésta sólo puede disminuirse mediante la toma de decisiones de incursionar en los mercados externos y el aprendizaje y compromiso que pueden resultar de esta experiencia concreta. Con ello, se iniciaba una perspectiva dinámica que la hacía diferente a las propuestas convencionales sustentadas en las teorías del comercio internacional.

Según Rialp (1999) este modelo explica la internacionalización como un proceso de desarrollo gradual que llevan a cabo las empresas en distintas fases durante un periodo relativamente largo, marcado por el compromiso creciente de vinculación con los mercados exteriores. Se hace énfasis en el aprendizaje obtenido y en el control de las variables organizacionales, así como en la capacidad de análisis del entorno y de su impacto en cada momento del tiempo. Se trata de acrecentar permanentemente los recursos físicos y de información destinados a este propósito, de tal forma que la exportación se convierta en una experiencia de aprendizaje en el ámbito internacional (Root, 1994). Alonso y Donoso (1994) concluyen, así, que las variables más influyentes en el proceso de internacionalización son el conocimiento del entorno internacional, el aprendizaje y la percepción sobre los mercados externos. En conclusión, los factores más relevantes a la hora de explicar la internacionalización son el conocimiento de los mercados externos y el compromiso adquirido con ellos.

Los autores coinciden en destacar como uno de los aportes más relevantes del modelo de Uppsala su consideración sobre el carácter incremental del proceso de aprendizaje, la caracterización de las distintas etapas sucesivas y el análisis de los principales obstáculos que pueden impedir una inserción exitosa en los mercados internacionales (Andrés y Salvaj, 2011). Así, el proceso de internacionalización se describe como una serie de etapas sucesivas que se denominan cadena de establecimiento en un mercado externo (Rialp 1997) y que suponen una progresiva 
profundización de las relaciones con el mercado externo y la asignación de mayores recursos a esta actividad, así como la adquisición de información valiosa que permite hacer sostenible en el tiempo el proceso. En la primera etapa la empresa realiza exportaciones esporádicas e irregulares, casi siempre de manera indirecta. Se inicia el proceso con escasa información sobre el mercado destino y la característica principal de esta etapa es la baja inversión hacia el conocimiento del mercado; el objetivo de esta primera fase es la adquisición del conocimiento sobre este mercado. Hasta tanto no se logre suficiente información sobre dicho mercado, no se puede avanzar a etapas posteriores.

Según los autores reseñados, en la segunda etapa la organización establece negocios internacionales a través de representantes independientes o agentes comercializadores, a través de los cuales obtiene información del mercado. Se caracteriza esta fase porque la empresa ya realiza esfuerzos notables para acceder al conocimiento del mercado que la lleven a consolidar las exportaciones con el objetivo de ejercer un mayor control sobre las ventas. Cuando ya se logra suficiente conocimiento del mercado y control total sobre las ventas y sobre el proceso de exportación, se da la tercera etapa que es el establecimiento de empresas filiales en el mercado externo o licencias internacionales para la venta del producto. La característica de esta tercera etapa es el control de las ventas y la amplia información que se tiene del comportamiento del mercado. El objetivo de esta etapa es adquirir suficiente experiencia sobre el mercado destino, de tal forma que se puedan establecer diferencias con los competidores.

En una fase posterior, la empresa ubica plantas de producción en el mercado externo; es decir, realiza inversión en el mercado extranjero a través del establecimiento de unidades productivas en dichos mercados, que le lleva a controlar las ventas y la producción y obtiene información directamente del mercado que se va a ver reflejada en las características del producto. Esta etapa está caracterizada por el control que se tiene de las ventas, de la producción y de la información sobre los factores de producción del mercado externo, factores todos que se utilizan para posicionar los bienes y servicios que se ofrecen en esos mercados. Esta cuarta etapa se considera eventual, ya que muchas empresas no logran, ni tienen como objetivo, llegar a establecer su producción en países externos.

El modelo inicialmente propuesto por Johanson y Wiedersheim-Paul (1975) fue ampliado posteriormente por Johanson y Vahlne (1977 y 1990) quienes explicaron la dinámica de internacionalización de la empresa no sólo en función del conocimiento del mercado y del proceso exportador sino en articulación con el compromiso creciente de recursos. Adicionalmente, le otorgan mayor relevancia al momento inicial del proceso, en el sentido de considerar el grado de acercamiento al mercado cuando se plantea como estrategia la internacionalización, como un elemento explicativo del ritmo y el éxito de la misma. Villarreal, a quien hemos citado anteriormente, considera relevante este complemento, ya que enfatiza en los aspectos dinámicos del proceso, que corresponden a decisiones de asignación de recursos y al desarrollo 
de actividades estratégicas para controlar el proceso de exportación y no sólo a los aspectos estáticos como son el conocimiento del mercado exterior y el compromiso con la actividades de expansión. (Villareal L, 2006).

En la perspectiva de Johanson y Vahlne (1990) los cambios organizacionales, la asignación de recursos a la preparación del recurso humano y la innovación en los programas de marketing, entre otros, reflejan el compromiso creciente con los mercados externos y se convierten en experiencia acumulada y aprendizaje valioso que facilitan el acceso a etapas más maduras como la inversión directa en el extranjero. Sin embargo, estos autores insisten en que esa experiencia es, de algún modo, específica para cada mercado, por lo que no se puede caer en el error de considerar que la conquista de un país es garantía de éxito en todos los demás, y mucho menos cuando se trata de culturas diferentes, preferencias del consumidor y estructuras político-económicas diversas. Lo anterior conduce a que se debe tener conocimiento, no sólo general sino específico sobre el mercado y sobre el proceso de exportación. Al hacer exportaciones a un mercado se adquiere conocimiento sobre el proceso mismo que puede transferirse cuando se quiere exportar a otros mercados, pero se requiere de un conocimiento específico sobre el mercado particular para aprovechar sus oportunidades y dinamismo y para conocer el carácter y la fuerza de los competidores. Es evidente que ese conocimiento sólo se adquiere mediante la presencia efectiva en el mercado, a través de la oferta de bienes o servicios específicos. Vale la pena insistir en que, para los teóricos suecos, cada fase exige mayores capacidades estratégicas y financieras por parte de las organizaciones puesto que el compromiso aumenta con el tiempo y, adicionalmente, son mayores los riesgos en los que se incurre

Otro elemento importante que destaca el modelo Uppsala tiene que ver con la variable que determina la selección de los mercados destino, que es lo que sus proponentes llaman distancia psicológica, que es la manera como el país de origen percibe en términos culturales al país destino, Johanson y Wiedersheim-Paul (1975) la definen como el conjunto de factores diferenciadores en aspectos como idioma, educación, economía, estructura de la industria, instituciones y política. De acuerdo a esto, para el país de origen son cercanos psicológicamente aquellos países que tengan alta similitud en dichos factores y lejanos los que muestran grandes diferencias. Los países prefieren, en sus fases iniciales de internacionalización, exportar a aquellos países que son psicológicamente más cercanos, porque el flujo de información es mucho más rápido y comprensible y, por tanto, se disminuye la incertidumbre en los negocios. Las exportaciones a países psicológicamente más distantes se hacen después que se haya adquirido el conocimiento sobre el proceso y se tenga información objetiva sobre dicho mercado.

Queremos destacar otra característica de este modelo, mencionada por algunos autores como Lamb y Liesch (2002) que resulta fundamental para la investigación adelantada sobre el sector exportador del AMB y es que, el compromiso con los mercados externos debe entenderse no sólo como la asignación de recursos para 
acceder a ellos sino también como la adecuación de actitudes y toma de decisiones empresariales que promuevan permanentemente este proceso. Para decirlo en palabras de Rialp (1999: p 7)

"Una aportación adicional radica en enfatizar la importancia de la percepción de oportunidades y riesgos por parte de los directivos de la empresa y, por tanto, de las capacidades y actitudes del equipo directivo ante la toma de decisiones internacionales".

Este elemento es importante, debido a las diversas interpretaciones sobre el desarrollo de nuestro departamento que le asignan a los directivos del sector empresarial una mentalidad conservadora y una fuerte aversión al riesgo, que se convierten, según algunos analistas, en obstáculos para alcanzar los niveles de competitividad exigidos por el ambiente internacional (Porter, 1994).

Vale la pena comentar que el modelo de Uppsala ha recibido críticas en los últimos años, especialmente a raíz de las dinámicas de industrialización acelerada de algunos países que se han insertado positivamente y en tiempo record en la economía globalizada. El principal cuestionamiento proviene, entonces, de la comprobación empírica de que, en algunos casos, el proceso gradualista de la escuela de Uppsala no se cumple, cuando las organizaciones emprenden tempranamente su vinculación con los mercados externos, a través de convenios de cooperación o alianzas estratégicas, por lo que podría decirse que se "saltan" algunas de las fases previstas en el modelo. Según Pla y Suárez (2001) los mismos autores que formularon la propuesta original, consideran la posibilidad de comportamientos diferenciados que no siguen las escalas sucesivas, especialmente cuando se tienen recursos suficientes como para ir más rápido o cuando los mercados son homogéneos y estables y es posible adquirir conocimiento sobre ellos por caminos distintos a la experiencia propia. Es necesario decir, sin embargo, que la existencia de excepciones no desvirtúa la perspectiva del análisis, cuyo principio fundamental es el aprendizaje incremental en la relación con los mercados externos, especialmente cuando se trata de empresas pequeñas y medianas, lo cual es aplicable, sin ninguna duda, a empresas que se inician en el diseño de esta estrategia, como es el caso de las PYME del AMB, nuestro objeto de estudio.

\subsection{El enfoque del Ciclo de Vida del Producto}

Otro modelo explicativo del proceso de internacionalización es el llamado del ciclo de vida del producto de Vernon (en adelante CVP), el cual representa también una visión evolutiva del proceso de la internacionalización empresarial. Según Villareal (2006), este modelo se basa en una conjunción de las teorías de las ventajas comparativas del comercio internacional con las teorías de decisión de inversión de las empresas articuladas, a su vez, con las teorías económicas que sustentan que los factores de producción son factores-clave en la internacionalización de las 
empresas. Vernon (1966) sugiere que la competitividad internacional, para cualquier tipo de industria, no es constante a los largo del tiempo y propone la visión de un producto que surge a partir de un proceso de innovación y atraviesa por cuatro etapas: introducción al mercado, crecimiento, madurez y declive.

La etapa de introducción se hace en el mercado doméstico, al aprovechar las oportunidades de la innovación del producto, derivadas de la demanda que encuentran en el mercado interno, ya sea por un mayor poder adquisitivo o porque los desarrollos tecnológicos inducen nuevas pautas de consumo. El autor mencionado considera que en países con altos ingresos promedio, en la fase de crecimiento el producto es habitual en el mercado innovador, se hacen los ajustes tecnológicos respectivos a partir de un seguimiento a los consumidores, por lo que se debe tener cercanía entre los consumidores y los puntos de fabricación del producto, al igual que en la fase anterior. En esta etapa aparecen los primeros esfuerzos de salida del producto hacia mercados externos en la forma de exportaciones coyunturales y, si las condiciones lo permiten, se trasladan etapas parciales o totales de fabricación a otros países. Al final de la misma, desaparecen las ventajas derivadas de la innovación y se centra la competencia en precios y calidad del producto.

En la etapa de maduración del producto se establece una ampliación entre la oferta y la demanda. Desaparece la ventaja de la primera empresa productora y se incrementa la competencia entre las empresas fabricantes del producto, paralelamente a un aumento de la demanda del producto. La competencia se centra en precios y en diferenciación, por lo que las empresas deben reducir drásticamente los costos de producción. Se incrementan las exportaciones del producto hacia países menos desarrollados y también, en algunos casos, ocurre el traslado de operaciones de fabricación a países donde las condiciones son más favorables en disposición y costos de mano de obra. La etapa de declive se presenta cuando el producto ya no es atractivo para los consumidores en el país de fabricación y/o en los países desarrollados. La producción y las ventas se centran, por tanto, en países menos desarrollados y la competencia internacional se define por los costos. La poca demanda que pueda existir en el país de origen del producto se cubre, igualmente, mediante importaciones de los países menos desarrollados en los que se concentra la producción en esta última fase del ciclo.

Según la versión del CVP, entonces, las empresas inician sus actividades económicas al destinar la producción al mercado local, en el cual adquieren cierto monopolio derivado de la innovación que desarrollan e imprimen a sus productos. Este grado de innovación se convierte en elemento diferenciador que les permite llegar a mercados extranjeros con ciertas ventajas tecnológicas. Estos mercados externos deben tener, inicialmente, características, estructuras y patrones de consumo similares al país de origen, para que gocen durante un tiempo de la ventaja innovadora, mientras sus competidores alcanzan desarrollos tecnológicos similares (López, 2006). La exportación a estos países con pautas y necesidades similares 
permite un aumento en la producción, para enfocarse a mercados de un mayor desarrollo industrial.

De acuerdo con Vernon (2006) las empresas deciden exportar a países que les representen ventajas, en términos de eficiencia económica, costos y rendimiento. Para seleccionar los mercados externos, se tienen en cuenta indicadores como patrones de demanda, economías de escala y barreras arancelarias, entre otras. Otras variables consideradas para definir la localización adecuada, tienen que ver con la elasticidad de la demanda, las características y la fuerza de los competidores en el mercado destino, así como el comportamiento del mercado, los costos y las características de la mano de obra.

Aunque este enfoque se centra en elementos endógenos a la empresa que es, en últimas, la que genera la innovación, algunos estudiosos consideran que se enfoca más en aspectos macroeconómicos, (Suárez y Galván, 2005) ya que es el entorno el que posibilita a las empresas alcanzar elevados grados de innovación. Estos elementos se ven reflejados en la estrategia de localización de la empresa y en el desarrollo del producto. Según la fase de desarrollo tecnológico en que se encuentre el producto, la empresa decide localizarse en cierta región que le representa disminución de costos y mayor desarrollo de tecnología, entre otros. En ese sentido, se integran elementos importantes para la internacionalización con la localización de la empresa, de acuerdo a la fase de desarrollo o madurez del producto (Pla y Suárez, 2001). Como consecuencia directa de esta dinámica de expansión internacional y desarrollo tecnológico, resulta el fenómeno de ubicación de plantas en lugares que brinden mayores condiciones para la generación del producto.

El modelo explicativo de la internacionalización basado en el CVP tiene como referente el escenario de los Estados Unidos, caracterizado por la presencia de grandes empresas multinacionales y un desarrollo tecnológico de avanzada (Durán J. J. 2005) lo que permite deducir que tiene validez en este contexto, es decir, para grandes empresas innovadoras y dentro de territorios altamente favorables para la innovación, que definen a los llamados países desarrollados. Así las cosas, puede considerarse como limitada su utilidad cuando se trata de analizar el comportamiento de pequeñas y medianas empresas (PYMES) en entornos en los que, los procesos de innovación son reducidos, como es el caso de Colombia y, particularmente, del Área Metropolitana de Bucaramanga.

\subsection{La Teoría de Recursos y Capacidades de la Organización}

Una de las teorías que más auge ha tenido en la última década establece que el comportamiento de las empresas en los mercados internacionales se puede explicar por los factores internos a la misma, especialmente, por las capacidades y recursos que poseen, unidos a la decisión de exportar y a la adopción de formas de exportación que les permitan obtener las ventajas competitivas necesarias para posicionarse en 
esos contextos. Esta visión estratégica que enfatiza en los factores internos a la empresa, por oposición a las que insistían en el análisis del impacto del entorno empresarial, se consolida en los años ochenta del siglo pasado y ha tenido múltiples desarrollos (Barney, 1991, Claver y Quer(2001).

El modelo da especial relevancia a las capacidades organizacionales que favorecen el proceso exportador y las considera un activo empresarial valioso y difícil de imitar por parte de los competidores. A nuestro juicio, tal como afirma Monreal (2009: p 8):

"este enfoque encaja en la dinámica del proceso de internacionalización en mercados turbulentos y de gran intensidad competitiva, al vincular la implicación de la empresa en los mercados externos al desarrollo de recursos y capacidades que sean compatibles con el mercado de exportación y con los objetivos de la empresa".

Los autores coinciden en señalar que, uno de los grandes retos a los que se enfrentan quienes pretenden orientar los estudios empíricos a partir de este enfoque, consiste en proponer una adecuada definición de capacidades y establecer cuáles son las más relevantes, desde el punto de vista estratégico, para la construcción de ventajas competitivas. En general, y según el autor que citamos en este párrafo, se asumen como capacidades las habilidades y rutinas organizacionales, los activos, procesos, características de la empresa, información y conocimiento que le permiten diseñar estrategias adecuadas para competir internacionalmente. Las propuestas destacan aquellas capacidades dinámicas que, superadas las que son exigidas para el cumplimiento de su misión, permiten a las organizaciones adaptarse fácilmente al cambio y renovarse permanentemente para ajustarse a los contextos complejos y cambiantes de la actualidad. Estas capacidades pueden darse en el campo de la producción, el marketing y la dirección empresarial.

La dotación de recursos y capacidades determina la competitividad internacional de la empresa de tres maneras: (a) porque la empresa posee un exceso de recursos y capacidades que pueden ser aprovechadas en el marco de mercados imperfectos; (b) la empresa puede transferir recursos y capacidades al mercado internacional en la forma de inversión directa extranjera y (c) porque la empresa posee una diferenciación en las capacidades y conocimientos intangibles, los mismos que, al combinarse con capitales físicos se traducen en una mayor competitividad de la empresa.

De este marco conceptual surgen los enfoques de modo de entrada a los mercados internacionales. Bastante aceptación ha tenido la propuesta de Alonso y Donoso (1991), en un gran estudio inicial sobre la competitividad de la empresa exportadora española, que ha dado pie para otras investigaciones en la misma línea en España. En dicho estudio, se proponen dos vías de internacionalización, una basada en la comercialización y otra en la producción, a la que se puede llegar a partir de la primera fase ymediante un proceso gradual.

Alonso y Donoso consideran que, para ingresar a mercados internacionales, la 
empresa requiere asumir un riesgo y un compromiso de recursos, que van unidos al control que pueda tener sobre el proceso de internacionalización. Como se dijo atrás, la exportación puede ser indirecta, por vía de la comercialización, o directa por vía de la producción ${ }^{3}$. La primera requiere un bajo compromiso de recursos por parte de la empresa y el riesgo que se asume es mínimo, pero supone muy poco control sobre el proceso de exportación. A medida que se compromete con formas de exportación directa, la organización debe invertir mayor cantidad de recursos, asumir riesgos mayores y, en contrapartida, tendrá un mayor control sobre el proceso de exportación. El trabajo de estos dos autores españoles enfatiza, adicionalmente, en que, las capacidades para triunfar en el mercado doméstico, difieren de las que se requieren para posicionarse competitivamente en los mercados internacionales.

Este enfoque se puede esquematizar con la Gráfica 1, presentada por los autores precitados:

Gráfica 1. Evolución modos de operación en mercados internacionales

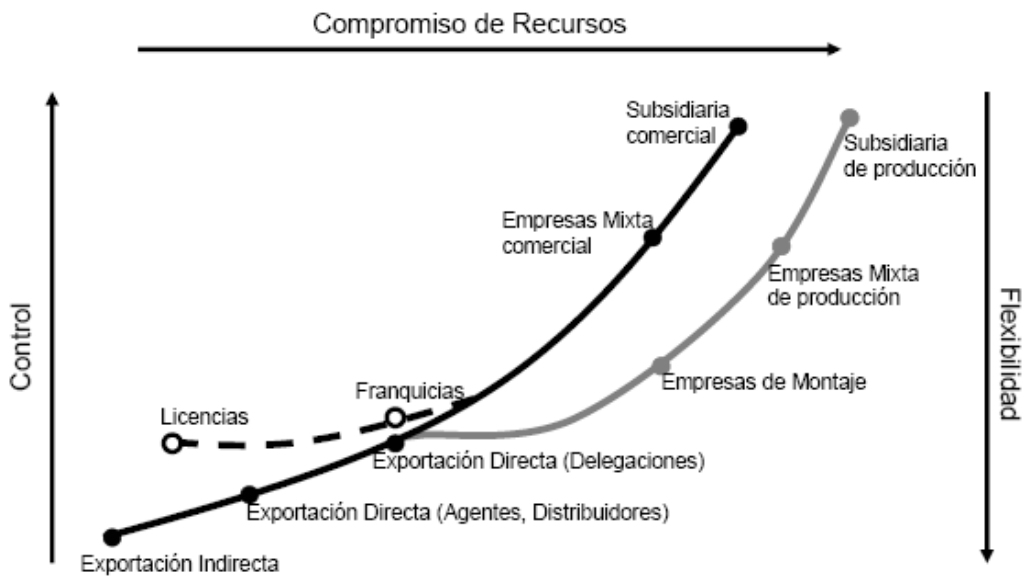

Riesgo

Fuente: Alonso y Donoso (1994)

3 La exportación es directa cuando la empresa se encarga no sólo del proceso de gestión de la exportación sino inclusive de distribuir el producto entre los consumidores finales en el país de destino. Las exportaciones son indirectas cuando el productor local no se ocupa de los trámites y procedimientos de exportación, en tanto que las exportaciones directas pueden efectuarse también bajo la modalidad de la subcontratación, en especial en el caso de las pequeñas empresas de los países en desarrollo. Dentro de la internacionalización comercial, se tienen empresas que exportan mediante grupos organizados o consorcios de exportación. Esta forma específica de internacionalización implica que la empresa se asocia con otras para poder efectuar actividades de exportación. 
Es importante anotar que, en años recientes, diversos estudiosos han concentrado su interés en el análisis de aquellas empresas nuevas que se insertan en muy corto tiempo en los mercados internacionales y que, por ese motivo, han sido denominadas bornglobals. En algunos ocasiones, se ha llegado a afirmar que esos casos exitosos le quitan piso a los enfoques gradualistas como los que hemos reseñado en el documento. Sin embargo, nuestro acercamiento a la realidad empresarial colombiana $y$, concretamente a la del departamento de Santander ${ }^{4}$ y el AMB, nos permite reafirmar la validez de esa visión de la internacionalización que supone la consolidación en los mercados locales como paso previo a las experiencias internacionales, y la superación de fases caracterizadas por bajos niveles de profundización y de riesgo, sin desconocer la presencia en nuestro medio de algunos casos de internacionalización acelerada (Plá y León, 2004).

\section{Las Pyme exportadoras del Área Metropolitana de Bucaramanga (AMB)}

En todo el mundo las pequeñas y medianas empresas han recibido en los últimos tiempos la atención de los estudiosos, debido al éxito obtenido por este tipo de organizaciones en procesos recientes de industrialización, por su reconocida flexibilidad para adaptarse con mayor facilidad a las realidades cambiantes del entorno actual y su capacidad para la generación de riqueza y empleo. Según Rodríguez (2003, p 28)

"Colombia es un país cuya economía se soporta en empresas de pequeña escala. Las PYME, conjuntamente con las microempresas, representan al menos el 90 por ciento del parque empresarial nacional y generan el $73 \%$ del empleo y el $53 \%$ de la producción bruta de los sectores industrial, comercial y de servicios".

A la vez, son la principal fuente de puestos de trabajo del país al emplear el 83,5\% de la población ocupada. Se ha reconocido también que son estas organizaciones las que presentan mayor dinamismo, en lo que se refiere a vinculación a los mercados internacionales y aprovechamiento de los tratados firmados por Colombia en los últimos tiempos, por lo que el Banco Mundial las reconoce como la columna vertebral de la economía.

$4 \quad$ El grupo de investigación ha realizado otras investigaciones sobre el proceso exportador de empresarios del Departamento de Santander y del Área Metropolitana de Bucaramanga: Proceso exportador de calzado de Santander a Ecuador y Venezuela (2009); y Análisis del proceso exportador de empresas del sector calzado y confecciones de Santander a Estados Unidos (2010) disponibles en la revista LEBRET 1 y 2 respectivamente. En ellos se confirma la tendencia de las empresas exportadoras a consolidarse primero en los mercados locales para luego buscar estrategias de expansión internacional. 
Sin embargo, la participación porcentual de las empresas de menor tamaño en las exportaciones del país es todavía muy baja, ya que en el 2005 no llegaba a una quinta parte del total, según el censo económico adelantado por el $\mathrm{DANE}^{5}$ en ese año. En un estudio reciente se afirma:

"las empresas grandes que representaban el 12\% de las empresas exportadoras, generaban el $80 \%$ de las ventas de manufacturas de Colombia, mientras que las PYMES que representaban la mitad de las empresas exportadoras efectuaron sólo el 11\% de las exportaciones" (Correa y otros, 2010, p 14).

Es posible que esa participación haya aumentado en los últimos años, como resultado de los distintos programas encaminados a mejorar la competitividad del sector productivo, aumentar la oferta exportable y diversificar el destino de la misma, pero sin que ello represente un cambio significativo en el panorama exportador a nivel nacional.

Para el departamento de Santander y el Área Metropolitana de Bucaramanga, el peso específico de las MIPYME es un poco mayor que en las capitales más grandes, por lo que resulta de especial importancia avanzar en el análisis de la internacionalización de este tipo de organizaciones, con el fin de establecer las tendencias relevantes, los alcances y los mayores obstáculos que ha enfrentado el sector productivo en su propósito de insertarse eficientemente en el complejo contexto internacional. De alguna forma, este tipo de estudios permite también hacerse una idea sobre la efectividad de las políticas estatales y las estrategias concertadas con el sector privado que han privilegiado la búsqueda de competitividad internacional. En este último punto, es importante recordar que, en el año 1999 se formuló el "Plan Estratégico Exportador 1999- 2009" y se creó el programa Expopyme, que ha beneficiado a una gran cantidad de empresas. Igualmente, el gobierno colombiano ha aprobado la Política Nacional de Competitividad y Productividad ${ }^{7}$ que da lineamientos para transformar la estructura productiva del país y alinearla con las tendencias internacionales, al mismo tiempo que reconoce, en el ejercicio prospectivo realizado con motivo de la conmemoración del segundo centenario de la independencia de Colombia ${ }^{8}$, la necesidad de reorientar los patrones de crecimiento del país, para que las PYMES se conviertan en un verdadero motor de desarrollo y generación de empleo.

5 Departamento Administrativo Nacional de Estadística de Colombia

6 "El Plan Estratégico Exportador fue concebido entre el sector privado, el sector público y la academia en el año 1999, como una estrategia de largo plazo tendiente a establecer las bases competitivas para incrementar la productividad y hacer de las exportaciones el motor de crecimiento de la economía, generando de esta forma un mayor desarrollo para el país, contribuyendo al mejoramiento de la calidad de vida de los colombianos": presentación del Ministerio de Comercio, Industria y Comercio de Colombia, (www.mincomercio.gov.co)

7 Documento CONPES, 3527 del 23 de junio de 2008

8 Departamento Nacional de Planeación (2005) Visión Colombia II Centenario: 2019 
Aún a pesar del dinamismo positivo registrado en la última década en algunos indicadores económicos y sociales del departamento de Santander y del AMB, la incursión en los mercados internacionales del sector productivo puede considerarse excesivamente tímida. Según la Cámara de Comercio Bucaramanga, menos del 3\% de las empresas de la ciudad han exportado en los últimos dos años. Ese pequeño subsector exportador se constituyó en el objeto de estudio de nuestra investigación. Así, se definió una muestra representativa a partir de los datos registrados en la base de datos Legiscomex, es decir, con empresas formalmente constituidas como tales y utilizado como criterio de selección el registro de exportaciones en el año anterior a la realización del estudio (2010). Para la selección de las empresas que finalmente se incluyeron en el estudio influyó, además de los criterios de inclusión, la aceptación y disponibilidad de sus directivos para suministrar información. Este hecho imposibilitó la utilización de una muestra estratificada por sector industrial, como se había planeado al inicio del proyecto.

El enfoque conceptual de la Escuela de Uppsala nos sirvió como referente básico para la definición de las variables a considerar en el análisis. Por ello, se toman como variables la experiencia internacional de las empresas, la característica de los mercados destino en función de su complejidad o distancia psicológica; la diversificación de mercados a los cuales se exporta; las formas de internacionalización y el conocimiento de los mercados donde se ubican sus productos. El estudio se complementa con la consideración de otras variables, como las actividades de preparación de las empresas para la exportación y los objetivos que se buscan con la internacionalización. En el Cuadro 1 se resumen las variables consideradas:

Cuadro 1. Definición de variables

\begin{tabular}{|c|c|c|}
\hline Variables & Interpretación & Parámetros \\
\hline $\begin{array}{l}\text { Experiencia } \\
\text { Internacional }\end{array}$ & $\begin{array}{l}\text { Tiempo que la empresa } \\
\text { ha realizado procesos } \\
\text { de exportación, ya sea } \\
\text { de manera esporádica o } \\
\text { de manera continua. }\end{array}$ & $\begin{array}{l}\text { Tiempo de presencia en los mercados } \\
\text { externos } \\
\text { - Años en los que realiza exportaciones de } \\
\text { manera continua. } \\
\text { - Tiempo de presencia en el principal } \\
\text { mercado }\end{array}$ \\
\hline $\begin{array}{l}\text { Característica del } \\
\text { mercado destino }\end{array}$ & $\begin{array}{c}\text { Expresado en función } \\
\text { de la distancia } \\
\text { psicológica de los } \\
\text { mercados destino, } \\
\text { que se derivada } \\
\text { de las similitudes } \\
\text { culturales, económicas, } \\
\text { productivas y sociales, } \\
\text { entre otras. }\end{array}$ & $\begin{array}{l}\text { Para las empresas del Área Metropolitana de } \\
\text { Bucaramanga los países psicológicamente } \\
\text { cercanos son los países latinoamericanos. } \\
\text { Los países psicológicamente lejanos son Estados } \\
\text { Unidos, Canadá y Países de Europa. }\end{array}$ \\
\hline
\end{tabular}




\begin{tabular}{|c|c|c|}
\hline $\begin{array}{l}\text { Diversificación } \\
\text { de mercados }\end{array}$ & $\begin{array}{c}\text { Número de mercados a } \\
\text { los cuales exportan las } \\
\text { empresas }\end{array}$ & $\begin{array}{l}\text { - Únicamente a un país } \\
\text { - Entre } 2 \text { y } 4 \text { países } \\
\text { - Entre } 5 \text { y } 7 \text { países } \\
\text { - Entre } 8 \text { y } 10 \text { países } \\
\text { - Más de } 10 \text { países }\end{array}$ \\
\hline $\begin{array}{l}\text { Formas de } \\
\text { interna- } \\
\text { cionalización }\end{array}$ & $\begin{array}{l}\text { Diversas maneras que } \\
\text { utilizan las empresas } \\
\text { para realizar el proceso } \\
\text { de ingreso y presencia } \\
\text { en los mercados } \\
\text { externos }\end{array}$ & $\begin{array}{l}\text { - } \text { Comercializadoras Internacionales } \\
\text { - Agentes y/o Distribuidores } \\
\text { - } \text { Faquila } \\
\text { - Verias } \\
\text { - Alianzas estratégicas extranjeras } \\
\text { - Alianzas con empresas colombianas } \\
\text { - Licencias de producción } \\
\text { - Puntos de venta propios en mercados } \\
\text { - } \text { Franquicias } \\
\text { - Plantas de producción en otros países }\end{array}$ \\
\hline $\begin{array}{l}\text { Actividades de } \\
\text { preparación para } \\
\text { la exporta ción: }\end{array}$ & $\begin{array}{l}\text { Conjunto de acciones } \\
\text { internas que realizan } \\
\text { las empresas para } \\
\text { dar mayor dinámica } \\
\text { al proceso de } \\
\text { internacionalización }\end{array}$ & $\begin{array}{l}\text { - Certificación de calidad } \\
\text { - Página Web } \\
\text { - Departamento de Comercio Exterior } \\
\text { - Investigación y desarrollo de productos } \\
\text { Plan exportador }\end{array}$ \\
\hline $\begin{array}{l}\text { Conocimiento del } \\
\text { mercado destino }\end{array}$ & $\begin{array}{l}\text { Nivel de apropiación } \\
\text { de variables } \\
\text { relacionadas con el } \\
\text { ingreso, exigencias y } \\
\text { comportamiento del } \\
\text { mercado destino }\end{array}$ & $\begin{array}{l}\text { - Requisitos de entrada } \\
\text { - Competencia } \\
\text { - Estructura de precios } \\
\text { - Canales de distribución } \\
\text { - Consumidor o cliente final } \\
\text { - Recesidades del mercado } \\
\text { - Contactos }\end{array}$ \\
\hline $\begin{array}{l}\text { Propósitos } \\
\text { de la interna- } \\
\text { cionalización }\end{array}$ & $\begin{array}{l}\text { Objetivos que han } \\
\text { llevado a la empresa a } \\
\text { internacionalizarse }\end{array}$ & $\begin{array}{l}\text { - } \text { Expansión de la empresa } \\
\text { - } \text { Opontactos establecidos } \\
\text { - } \text { acuerdos comerciales } \\
\text { - Información sobre oportunidades en } \\
\text { - } \text { Planeación estratégica } \\
\text { - } \text { Diversificar riesgos de las operaciones } \\
\text { - Obtener economías de escala } \\
\text { - Promoción de instituciones } \\
\text { - Estabilizar las ventas de productos } \\
\text { - } \text { (mercado doméstico estacional) } \\
\text { - } \text { doméstico } \\
\text { - Expas de cambio favorables } \\
\text { - } \text { que pertenece la empresa está exportando } \\
\text { - Excedentes de producción }\end{array}$ \\
\hline
\end{tabular}




\section{Internacionalización, distancia psicológica y conocimiento de los mercados}

Una primera aproximación a las empresas exportadoras del AMB demostró que su presencia en los ámbitos internacionales es relativamente reciente y que son muy pocas las que han mantenido un ritmo constante en la actividad exportadora: la mitad de ellas tienen menos de diez años de haber incursionado en los mercados externos, en tanto que, en la otra mitad, sólo un 12,5\% lleva más de veinte años exportando y lo hace de manera continua, aunque un porcentaje mucho menor se ha mantenido en un mismo mercado-destino (Véase Cuadro 2) Estos datos ponen de presente un proceso de internacionalización todavía incipiente que no puede definirse aún como sostenible en el tiempo.

Cuadro 2. Experiencia Internacional de las empresa exportadoras del Área Metropolitana de Bucaramanga

\begin{tabular}{|l|c|c|c|}
\hline \multicolumn{1}{|c|}{ Rango } & $\begin{array}{c}\text { Presencia } \\
\text { internacional }\end{array}$ & $\begin{array}{c}\text { Exportación de } \\
\text { manera continua }\end{array}$ & $\begin{array}{c}\text { Presencia internacional en el } \\
\text { principal mercado }\end{array}$ \\
\hline & $\%$ & $\%$ & $\%$ \\
\hline 1 a 5 años & 21,9 & 21,9 & 28,1 \\
\hline 6 a 10 años & 28,1 & 37,5 & 34,4 \\
\hline 11 a 15 años & 18,8 & 12,5 & 12,5 \\
\hline 16 a 20 años & 18,8 & 15,6 & 18,8 \\
\hline Mayor de 20 años & 12,5 & 12,5 & 6,3 \\
\hline Total & 100 & 100 & 100,0 \\
\hline
\end{tabular}

Elaboración propia

El enfoque de Uppsala que nos sirve de referente, postula que las empresas inician sus contactos internacionales cuando su tamaño es reducido y los expanden progresivamente hacia los mercados psicológicamente más cercanos, es decir, aquellos con los que la empresa puede establecer flujos de información por una relativa similitud en aspectos culturales como el idioma, el sistema político, los niveles de educación o el desarrollo industrial Johanson y Vahlne (1977). Así, se concibe la distancia psicológica como el conjunto de factores que impiden que este proceso de interrelación se construya de manera fluida.

Este principio se cumple a cabalidad para el caso de las empresas del AMB, que muestran una tendencia clara a orientar sus exportaciones a los países 
latinoamericanos. El 71,8\% de las empresas exportadoras tiene como principales mercados a Ecuador y Venezuela, países fronterizos con Colombia y que siempre se han considerado mercados naturales, por los orígenes históricos compartidos y la similitud en las costumbres y pautas de consumo. El resto de las empresas tiene como principal mercado países anglosajones como Estados Unidos, Canadá e Inglaterra, que son considerados psicológicamente lejanos pero han tenido, a través del tiempo, relaciones económicas fuertes con Colombia y en algunos casos - como el de Estados Unidos- han ofrecido a nuestro país incentivos exportadores de importancia, como las preferencias arancelarias (Véase Cuadro 3).

Cuadro 3. Distancia psicológica de los mercados destino de las empresas exportadoras del Área Metropolitana de Bucaramanga

\begin{tabular}{|l|c|c|}
\hline Principal mercado & $\%$ & Distancia psicológica \\
\hline Ecuador & 31,3 & Cercano \\
\hline Venezuela & 15,6 & Cercano \\
\hline Estados Unidos & 12,5 & Lejano \\
\hline Canadá & 9,4 & Lejano \\
\hline Puerto Rico & 6,3 & Cercano \\
\hline México & 6,3 & Cercano \\
\hline Perú & 6,3 & Cercano \\
\hline Inglaterra & 6,3 & Lejano \\
\hline Antillas & 3,1 & Cercano \\
\hline Panamá & 3,1 & Cercano \\
\hline Total & 100,0 & \\
\hline
\end{tabular}

Elaboración propia

En frecuencia de destinos, el $90 \%$ de las empresas exporta a países latinoamericanos; el 50\% exporta a Estados Unidos, país considerado atractivo para las empresas del Área Metropolitana de Bucaramanga por el gran tamaño del mercado. El 15\% envía productos a países de la Unión Europea. No se registraron exportaciones a países de Asia, África y Oceanía por parte de las empresas participantes en el estudio, debido a la percepción generalizada de que son mercados difíciles y muy alejados, tanto en términos geográficos como culturales (Véase Cuadro 4). 
Cuadro 4. Área de mercado de exportación de las empresas del

Área Metropolitana de Bucaramanga

\begin{tabular}{|c|c|}
\hline Área de mercado de exportación & Frecuencia (\%) \\
\hline Latinoamérica & $90,6 \%$ \\
\hline Norteamérica & $50,0 \%$ \\
\hline Unión Europea & $15,6 \%$ \\
\hline
\end{tabular}

Elaboración propia

\subsection{Motivaciones y formas de la Internacionalización}

Como una demostración que los empresarios exportadores del Área Metropolitana de Bucaramanga son conscientes de la necesidad de adaptarse a las condiciones del entorno internacional, caracterizado por la interdependencia de las economías y la puesta en vigor de acuerdos comerciales de carácter bilateral o multilateral, la mayoría expresa que la vinculación al mercado exterior se concibe como una estrategia de largo plazo. Así, dentro de las principales razones que explican la búsqueda de internacionalización de la empresa, además de la expansión en términos de ventas, se encuentran el aprovechamiento de las oportunidades que ofrecen los acuerdos comerciales y la posibilidad de obtener conocimiento de los mercados externos y utilizar contactos previos existentes. Entre las razones que se ponderaron con menor importancia aparecen el aprovechamiento de las tasas de cambio favorables, exportar porque la competencia o la cadena productiva exportan o para dar salida a los excedentes de producción (Véase Cuadro 5).

Cuadro 5. Ranking de razones para la internacionalización entre los exportadores del Área Metropolitana de Bucaramanga

\begin{tabular}{|l|c|}
\hline Razones & Orden de importancia \\
\hline Expansión de la empresa & 1 \\
\hline Contactos establecidos & 2 \\
\hline Oportunidades identificadas en los acuerdos comerciales & 3 \\
\hline Información sobre oportunidades en mercados externos & 4 \\
\hline Planeación estratégica & 5 \\
\hline Diversificar riesgos de las operaciones & 6 \\
\hline Obtener economías de escala & 7 \\
\hline Promoción de instituciones & 8 \\
\hline Estabilizar las ventas de productos (mercado doméstico estacional) & 9 \\
\hline Situación coyuntural del mercado domestico & 10 \\
\hline
\end{tabular}




\begin{tabular}{|l|c|}
\hline Tasas de cambio favorables & 11 \\
\hline $\begin{array}{l}\text { Exportar porque la cadena productiva a la que pertenece la empresa } \\
\text { exporta }\end{array}$ & 12 \\
\hline La competencia doméstica exporta & 13 \\
\hline Excedentes de producción & 14 \\
\hline
\end{tabular}

Elaboración propia

Tal como se planteó atrás, el enfoque de Uppsala considera la internacionalización como un proceso lento y gradual que se inicia con exportaciones irregulares, se consolida con la instauración de filiales en el extranjero, y concibe cada fase como un paso adelante en términos de conocimiento de los mercados y asignación de recursos efectivos al proceso. Para el caso del AMB, la internacionalización se concreta únicamente en exportaciones, que son realizadas de manera directa o indirecta. No se evidenciaron otras formas de internacionalización como puntos de venta en mercados externos, plantas de producción en otros países, franquicias; licencias o consorcios con empresas extranjeras.

Las exportaciones las realizan de manera directa a través de agentes o distribuidores, por medio de ventas directas a clientes externos y, de manera indirecta, a través de comercializadoras internacionales. Se evidencia, además, que algunas empresas están insertas en cadenas de valor internacionales (maquila) y, por tanto, el proceso exportador adquiere el carácter de intra-industrial. La participación en ferias internacionales se constituye en otra forma de realización de exportaciones esporádicas. Un elemento positivo detectado es la tendencia, en la mayoría de las empresas, a realizar las exportaciones de manera directa, por lo que se está en la vía correcta para adquirir conocimiento de los mercados externos y del proceso mismo de exportación (Véase Cuadro 6).

Cuadro 6. Formas de internacionalización de las empresas del Área Metropolitana de Bucaramanga

\begin{tabular}{|l|c|}
\hline Formas de entrada & Frecuencia \% \\
\hline Agentes y/o Distribuidores & 50,0 \\
\hline Ventas directas a clientes externos y consumidores finales & 34,4 \\
\hline Comercializadoras Internacionales & 28,1 \\
\hline Maquila & 9,4 \\
\hline Presencia en Ferias & 3,1 \\
\hline Alianzas estratégicas extranjeras & 6,3 \\
\hline Alianzas con empresas Colombianas & 3,1 \\
\hline
\end{tabular}

Elaboración propia 


\subsection{Diversificación y conocimiento de los mercados externos}

Una característica notoria de las PYMES exportadoras del AMB es la escasa diversificación de los mercados a los que exportan, lo que las hace muy sensibles a aspectos coyunturales que pueden afectar la demanda. El 19\% sólo tiene un mercado-destino; el 75\% exporta a menos de cinco países, el 22\% de las empresas tiene contactos con más de cinco países y sólo el 3\% tiene más de diez destinos para sus exportaciones. Estos hechos reflejan alta dependencia de los mercados a los cuales se exporta que se traduce, como ya se dijo, en alta sensibilidad a las dificultades de esos mercados. El impacto creciente del deterioro de las relaciones colombo-venezolanas durante el gobierno pasado, es un ejemplo concreto de esta situación que, sin embargo, pudo tener tangencialmente un efecto positivo, al incentivar la búsqueda de mercados-reemplazo, especialmente en Centro-América (Véase Cuadro 7).

Cuadro 7. Diversificación de mercados de las empresas exportadoras del Área Metropolitana de Bucaramanga

\begin{tabular}{|l|c|}
\hline & $\%$ \\
\hline Únicamente a un país & 18,8 \\
\hline Entre 2 y 4 países & 56,3 \\
\hline Entre 5 y 7 países & 12,5 \\
\hline Entre 8 y 10 países & 9,4 \\
\hline Más de 10 países & 3,1 \\
\hline Total & 100 \\
\hline
\end{tabular}

Elaboración propia

Como se expresó al inicio del artículo, el enfoque gradualista de la internacionalización establece una relación directa entre el conocimiento del mercado y el compromiso de recursos para avanzar en el proceso, razón por la cual la primera variable se convierte en fundamental en las primeras fases de articulación en los mercados internacionales. El estudio comprobó que la mayoría de las empresas exportadoras del Área Metropolitana de Bucaramanga tiene buenos conocimientos de los mercados a los cuales exportan, especialmente en lo que se refiere a requisitos de entrada, estructura de precios, necesidades del mercado y requerimientos de adaptación del producto. Se requiere afianzar conocimientos respecto a contactos en el mercado destino, posibles canales de distribución, características de la competencia y gustos y necesidades del consumidor final, factores estos últimos que se relacionan directamente con las estrategias de mercadeo internacional, que se constataron como muy débiles en el sector productivo estudiado (Véase Cuadro 8). 
Cuadro 8. Ponderación del conocimiento de los mercados externos por parte de los exportadores del Área Metropolitana de Bucaramanga

\begin{tabular}{|l|c|c|c|c|c|}
\hline Factores & $\begin{array}{c}\text { Ningún } \\
\text { conocimiento }\end{array}$ & $\begin{array}{c}\text { Bajo } \\
\text { conocimiento }\end{array}$ & $\begin{array}{c}\text { Conocimiento } \\
\text { medio }\end{array}$ & $\begin{array}{c}\text { Alto } \\
\text { conocimiento }\end{array}$ & Muy alto \\
\hline $\begin{array}{l}\text { Requisitos de } \\
\text { entrada }\end{array}$ & 0,0 & 0,0 & 12,5 & 56,3 & 31,3 \\
\hline $\begin{array}{l}\text { Estructura de } \\
\text { precios }\end{array}$ & 0,0 & 9,4 & 34,4 & 40,6 & 15,6 \\
\hline $\begin{array}{l}\text { Necesidades del } \\
\text { mercado }\end{array}$ & 0,0 & 18,8 & 28,1 & 31,3 & 21,9 \\
\hline $\begin{array}{l}\text { Requerimientos } \\
\text { de adaptación } \\
\text { producto) }\end{array}$ & 3,1 & 6,3 & 37,5 & 37,5 & 15,6 \\
\hline Contactos & 0,0 & 18,8 & 21,9 & 28,1 & 28,1 \\
\hline $\begin{array}{l}\text { Canales de } \\
\text { distribución }\end{array}$ & 3,1 & 18,8 & 21,9 & 34,4 & 21,9 \\
\hline Competencia & 0,0 & 15,6 & 46,9 & 28,1 & 9,4 \\
\hline $\begin{array}{l}\text { Consumidor o } \\
\text { cliente final }\end{array}$ & 0,0 & 31,3 & 15,6 & 43,8 & 9,4 \\
\hline
\end{tabular}

Elaboración propia

Reforzada la percepción de que las PYMES exportadoras del AMB han asumido el proceso de internacionalización como un objetivo estratégico, se comprobó que la mayoría de ellas diseñan estrategias para facilitar, de alguna forma, la inserción positiva en los mercados internacionales. Más del $80 \%$ ha implementado una página Web de su empresa para dar a conocer los bienes que ofrece y como mecanismo de contacto con los clientes externos. También las empresas dedican esfuerzos a programas de investigación y desarrollo de productos para presentar innovaciones en los mercados-destino. Aproximadamente la mitad de las empresas formulan planes exportadores y cuentan con departamentos de Comercio Exterior, que se dedican exclusivamente a la planeación y ejecución de las actividades de internacionalización. Así mismo, un buen número de empresas cuenta con certificaciones de calidad para dar mayor respaldo a sus productos en los mercados a los cuales exportan (Véase Cuadro 9). 
Cuadro 9. Actividades de preparación para la exportación realizadas por las empresas del Área Metropolitana de Bucaramanga

\begin{tabular}{|l|c|}
\hline Actividades & Frecuencia \\
\hline Página Web & 81,3 \\
\hline Investigación y desarrollo de productos & 71,9 \\
\hline Plan exportador & 54,8 \\
\hline Departamento de Comercio Exterior & 48,4 \\
\hline Certificación de calidad & 45,2 \\
\hline
\end{tabular}

Elaboración propia

\section{A manera de conclusión}

El estudio sobre el proceso de internacionalización de las PYMES manufactureras del Área Metropolitana de Bucaramanga, deja ver que no es posible encajar estrictamente el proceso dentro de los lineamientos de uno de los modelos conceptuales existentes, pero si evidencia la tendencia hacia el modelo gradualista, aunque con cierto grado de estancamiento, ya que no se denota evolución a fases posteriores. Este último punto debe constituirse, a nuestro juicio, en un punto de partida y un reto para futuras investigaciones sobre el tema.

Aún cuando los empresarios exportadores del Área Metropolitana de Bucaramanga tienden a exportar a mercados psicológica y geográficamente cercanos, se observan algunos casos de empresas que realizan exportaciones a mercados psicológicamente distantes, donde las diferencias culturales son marcadas y las exigencias de entrada complejas, por lo que puede resultar esclarecedor indagar sobre las razones del éxito de estas unidades empresariales. Para el equipo investigador, la visualización de los factores que han contribuido a este proceso, se plantea como una segunda etapa del proyecto, en la que se realice un análisis endógeno que privilegie la perspectiva de los recursos y capacidades organizacionales.

Por último, en momentos en que el gobierno y las entidades de apoyo y fomento a la internacionalización de la economía colombiana hacen esfuerzos por establecer acuerdos con la Unión Europea y con países asiáticos como Corea, se evidencia que las PYMES exportadoras del AMB tienen una insignificante participación en dichos mercados. Son pocas las empresas que exportan a países europeos y no se encontró evidencia de empresas dentro del sector manufacturero que realicen exportaciones al Oriente y Lejano Oriente. Por tal motivo, este es un tercer ámbito que puede resultar de interés para estudios posteriores, siempre y cuando se logre vencer la resistencia de las empresas regionales a hacer realidad la necesaria colaboración 
entre el sector productivo y las universidades con el fin de que no se pierdan los esfuerzos investigativos y se conviertan, en cambio, en esclarecedores de propuestas estratégicas que mejoren el desempeño competitivo de las empresas y jalonen los procesos de desarrollo local y regional.

\section{REFERENCIAS BIBLIOGRÁFICAS}

Alonso J. A. y Donoso V. (1994). La competitividad de la empresa exportadora española. ICEX. Madrid.

Andrés María F, y Salvaj Erica (2011). "La internacionalización acelerada en Pymes de reciente creación” I Congreso de la Red de Integración Latinoamericana 2011 Universidad Nacional del Litoral, Santafé Argentina. Consultado en: http://www. uncu.edu.ar/relacionesinternacionales/upload/redilaeje54.pdf

Banco Mundial 2010. Doing Business 2010 Colombia. En: http://espanol. doingbusiness.org/ExploreEconomies/?economyid=46; consulta: marzo de 2010.

Barney, J.B. (1991), Firm resources and sustained competitive advantage. Journal of Management, Vol 17 N. 1

Cámara de Comercio de Bogotá. 2009. Estructura empresarial. Bogotá.

Canals, Jordi (1996). La Internacionalización de la Empresa. Editorial McGraw Hill - IESE.Madrid.

Claver, Enrique y Diego Quer (2001). "La dirección estratégica de la internacionalización de la empresa: propuesta de un marco teórico integrador". Información Comercial Española, No. 794, pp. 37- 59.

Correa G. L.; Durán Y; Segura O. (2010), Redes empresariales para la internacionalización. FUNDES

DNP, Consejo Nacional de Política Económica y Social. 2008. Política nacional de competitividad y productividad. Documento CONPES 3527. Departamento Nacional de Planeación, Bogotá.

Daniels John y Radebaugh Lee (2000). Negocios internacionales. Octava edición. México. Pearson educación.

Durán, J. J. (2005): Teoría de la Economía y Dirección de la Empresa Multinacional: La Empresa Multinacional Española. Estrategias y Ventajas Competitivas, Minerva Ediciones, Madrid.

Galván, I. (2003), La Formación de la Estrategia de Selección de Mercados Exteriores en el Proceso de Internacionalización de las Empresas, Tesis Doctoral, Universidad de las Palmas de Gran Canaria, España. 
Johanson, J., y Vahlne, J.-E. (1977), The internationalization of the firm-A Model of knowledge development and increasing foreign market commitmnents. Journal of International Business Studies, Vol. 8 (1), pp. 23-32.

Johanson, J., y Vahlne, J.-E. (1990), The mechanism of internationalization, International Marketing Review, Vol. 7 (4).

Johanson, J., y Wiedersheim-Paul, F. (1975), The internationalization of the firm-four Swedish cases, Journal of Management Studies, Vol. 12.

Lamb P. y Liesch P. W. (2002), Re-framing the relationships between market commitment, knowledge and involvement, Management International Review, 42 (1).

López, José (2006). La internacionalización de la empresa manufacturera española: efectos del capital humano genérico y específico". Cuadernos de Gestión. No. 1, Vol. 6, pp. 11-24

Monreal J. P. (2009), Análisis del comportamiento exportador de la empresa española desde el enfoque de los recursos y las capacidades. Tesis doctoral Universidad de Murcia. Disponible en http://www.tesisenred.net/bitstream/handle/ 10803/11003/ MonrealPerez.pdf

Plá J y León, F. (2004), Dirección de Empresas Internacionales. Editorial Pearson Educación S. A. Madrid, España

Plá, J.B, y Suárez Ortega, S.M. (2001), ¿Cómo se explica la internacionalización de la empresa? Una perspectiva teórica integradora”, ICADE Revista de las Facultades de Derecho y Ciencias Económicas y Empresariales, Vol. 52 (Enero-Abril).

Porter, M. (1994), Informe Monitor. Creando la Ventaja Competitiva de Colombia. Disponile en: www.camaramed.org.co/docs/01informe_monitor_colombia.doc

Quer, Diego, Enrique Claver y José Molina (2006). Riesgo, distancia cultural y estrategia de entrada: nueva evidencia empírica en el proceso de internacionalización de la empresa española”. Boletín Económico del ICE. No. 2875, pp. 13-21.

Rialp, Alex (1999). Los enfoques micro-organizativos de la internacionalización de la empresa: una revisión y síntesis de la literatura”. Información Comercial Española. No. 781, pp. 117-128.

Rodríguez A. G. (2003), La realidad de la Pyme colombiana. Desafío para el desarrollo. FUNDES

Root, F.R. (1994), EntryStrategiesfor International Markets, Lexington Books, Nueva York. 
Simón Carrión R, (2010). Innovación, internacionalización y cooperación, un estudio de caso basado en el CTAP, Universidad de Almería (España)

Suárez, Sonia, Arístides Olivares e Inmaculada Galván (2002). La expansión de los mercados de exportación y el tamaño empresarial: el caso de las empresas exportadoras canarias”. Información Comercial Española. No. 802, pp. 83-97.

Velásquez V., F. (2004). La estrategia, la estructura y las formas de asociación: fuentes de ventaja competitiva para las Pymes colombianas. Estudios Gerenciales 093, 73-97.

Vernon, R. (1966), International investment and international trade in the product cycle, Quarterly Journal of Economics.

Villarreal L.O (2006) la estrategia de internacionalización de la empresa. Un estudio de casos de multinacionales Vascas. Tesis doctoral. Universidad del País Vasco. Bilbao.

Villarreal, Óskar (2005). La internacionalización de la empresa y la empresa multinacional: una revisión conceptual contemporánea”. Cuadernos de Gestión. No. 2 Vol. 5, pp. 55-73. 